\title{
Intestinal microsporidiosis: prevalence and genetic study of Egyptian isolates
}

Original

Article

\author{
Mohammad H Abd-Elbaki, Magdy A Arafa, Dina M Abd-El Hameed, Khaled S \\ Habib, Abeer A Abdel Rahman, Mona M Anwar
}

Department of Parasitology, Faculty of Medicine, Ain Shams University, Cairo, Egypt

\begin{abstract}
Background: Diagnosis of microsporidiosis is difficult due to its small size needing special stains and identification by an expert. Nowadays, application of polymerase chain reaction (PCR) made diagnosis more sensitive, specific and easier.

Objectives: To update the prevalence of intestinal microsporidia and to clarify their genotype patterns in symptomatic and asymptomatic immune compromised and immune competent cases.

Patients and Methods: Totally, 323 stool samples were collected and subdivided as 173 from immune compromised (group I) and 150 from immune competent (group II) individuals. Samples were examined for microsporidiosis by light microscopy smears stained by Weber's modified trichrome (WMT) and modified Ziehl Neelsen (MZN), as well as by nested and RFLP PCR techniques.

Results: Microsporidial spores were microscopically detected in 45/323 (13.9\%) individuals; 25/173 $(14.5 \%)$ immune compromised and 20/150 (13.3\%) immune competent cases. In the two groups, 25/45 (55.6\%) were symptomatic complaining of diarrhea, abdominal pain, distension, mal-digestion and weight loss, with statistical significant difference $(P<0.001)$ between infection and presence of symptoms. Nested and RFLP PCR missed only one positive case, thus scoring $97.8 \%$ sensitivity and $100 \%$ specificity, positive predictive value $100 \%$, negative predictive value $99.6 \%$ and diagnostic accuracy $97.8 \%$. In group I, 5 cases were associated with Enterocytozoon (Ent.) bieneusi, 5 with Encephalitozoon (Enc.) species and 15 had mixed infection. In group II, 6 had Ent. bieneusi, 3 had Enc. species and 10 had mixed infection. Sequencing of the internal transcribed spacer of rDNA of five samples demonstrated the Ent. bieneusi anthroponotic genotype $\mathrm{B}$ and the zoonotic potential for genotypes $\mathrm{D}$ and $\mathrm{K}$, in addition to one Enc. intestinalis sequence. Conclusion: Prevalence of microsporidiosis was insignificantly higher in immune compromised than immune competent population. Intestinal microsporidiosis can be manifested by different abdominal symptoms or it can be asymptomatic. PCR technique highlighted that mixed infection with Ent. bieneusi and Encephalitozoon species was the commonest finding among the studied groups. Ent. bieneusi genotypes appeared to be related to animal contact and human infection. This, however, could not be accurately defined due to the limited number of available sequences.
\end{abstract}

Keywords: Enc. intestinalis, Ent. bieneusi, genotypes, immune competent, immune compromised, microsporidia.

Received: 17 October, 2019, Accepted: 24 January, 2020.

Corresponding Author: Abeer A Abdel Rahman ,Tel.: +20 1001079495, E-mail: abeer1124@yahoo.com

Print ISSN: 1687-7942, Online ISSN: 2090-2646, Vol. 13, No. 1, April, 2020.

\section{INTRODUCTION}

Microsporidia are small, spore forming, obligate intracellular protozoa detected in many hosts. Up till now, 1300 microsporidian species or more, related to 150 genera, were detected ${ }^{[1]}$. Ent. bieneusi together with three species of genus Encephalitozoon, Enc. hellem, Enc. intestinalis, and Enc. cuniculi, were the main species discovered in HIV-infected patients ${ }^{[2]}$, renal transplants recipients ${ }^{[3]}$ and cancer patients ${ }^{[4]}$. The diagnosis is often missed as the spores of microsporidia are variably small in size ranging from 1-4 $\mu$. Earlier, transmission electron microscopy was considered the gold standard for diagnosis of intestinal microsporidiosis; however, it was found to be time consuming, expensive and not available in most settings ${ }^{[5]}$. Presently, laboratory diagnosis depends upon direct visualization of spores in fecal samples by light microscopy using WMT staining and Fluorescent microscopy using Calcoflour White staining technique, but both techniques cannot differentiate between the species ${ }^{[6]}$. Polymerase chain reaction is being widely used for species and genotype detection of microsporidia in immune compromised and immune competent individuals ${ }^{[5,7]}$. Observed variation among microsporidia isolates as a result of different internal transcribed spacer (ITS) gene region of rRNA, help in the discrimination between host-adapted and zoonotic genotypes, in addition to geographical distribution of these genotypes $^{[8]}$. Species differentiation has important therapeutic implications, as Enc. intestinalis and Ent. bieneusi respond variably to the currently used drug albendazole ${ }^{[9]}$. Although albendazole is highly active against all of the Encephaliozoonidae in vitro ${ }^{[10]}$, it is not very effective against Ent. bieneusi ${ }^{[11]}$. 
In the present study, we aimed to investigate the occurrence of intestinal microsporidiosis in immune compromised and immune competent individuals, and determine the most prevalent and pathogenic genotypes in Egypt. Prevalence was determined by examining stool smears stained by WMT and MZN stains. Genotypes in recorded cases were identified by nested and RFLP PCR techniques.

\section{PATIENTS AND METHODS}

Sample collection: In a case control study, a total of 323 stool samples were examined for intestinal microsporidiosis in the period from June 2017 to May 2018. One hundred and seventy three samples were from immune compromised patients (exposed to chemotherapy and/or radiotherapy, receiving corticosteroids due to organ transplant or autoimmune diseases, malnutrition in children and from elderly patients). They were chosen from patients admitted to the Chemotherapy, Radiotherapy, Hematology, Nephrology, Geriatric and Pediatric Departments, Faculty of Medicine, Ain Shams University. In addition, 150 stool samples were collected and examined from immune competent individuals attending Parasitology Research Unit, Medical Parasitology Department, Faculty of Medicine, Ain Shams University. A full history sheet was completed for each subject including age, sex, diagnosis, disease stage, treatments received as well as contact with animals. The immune compromised patients were selected from isolation sectors in the mentioned departments according to their estimated low $\mathrm{CD}+4$ counts and total leukocyte count of less than $4000 / \mathrm{m} 3$, in their investigation file.
Subject grouping: The study included 2 main groups: group I (173) immune compromised patients, and group II (150) immune competent individuals. Each group was subdivided into 2 subgroups: $a$ and $b$, according to presence or absence of GIT symptoms (diarrhea, abdominal distension, maldigestion). Groups Ia and Ib included 22 symptomatic and 151 asymptomatic immune compromised patients, respectively; while immune competent subgroups IIa and IIb included 15 symptomatic and 135 asymptomatic individuals, respectively. Patients were considered diarrheic if complaining of abnormal increase in stool fluency and/ or frequency of bowel motions.

Sample processing: Three consecutive stool samples were collected from each patient and control in properly labeled screw capped plastic containers and taken directly to the laboratory of Medical Parasitology Department, Faculty of Medicine, Ain shams University. Air dried stool smears were fixed with methanol, stained with WMT ${ }^{[12]}$ and MZN ${ }^{[13]}$ stains, and examined under light microscope (x1000). Only stool samples positive for microsporidia were aliquoted in sterile $2 \mathrm{ml}$ eppendorf tubes and stored in $-20^{\circ} \mathrm{C}$ for DNA extraction.

Molecular studies: DNA extraction from stool samples was performed using QIAamp-DNA stool minikit (Qiagen, Valencia, CA, USA) according to Sokolova et al. ${ }^{[14]}$. DNA was eluted and kept at $-20^{\circ} \mathrm{C}$. PCR amplification of the internal transcribed spacer (ITS) and flanking regions of the small subunit (SSU) and large subunit (LSU) of the ribosomal DNA (rDNA) were the targets for the nested-PCR procedure ${ }^{[14]}$. The following table shows the used primers.

\begin{tabular}{cllc}
\hline \hline Primers & & \multicolumn{1}{c}{ Sequence } & Identification \\
\hline \multirow{2}{*}{ Upstream } & MSP-1 & TGA ATG [G/T]GT CCC TGT & \multirow{2}{*}{ Microsporidium spp. } \\
& MSP-3 & GGA ATT CAC ACC GCC CGT C[A/G][C/T] TAT & \multirow{2}{*}{ Ent. bieneusi } \\
\hline \multirow{5}{*}{ Downstream } & MSP-2B & GTT CAT TCG CAC TAC T & \multirow{2}{*}{ Encephalitozoon spp. } \\
\cline { 2 - 5 } & MSP-4B & CCA AGC TTA TGC TTA AGT CCA GGG AG & TCA CTC GCC GCT ACT \\
& MSP-4A & CCA AGC TTA TGC TTA AGT [C/T][A/C]A A[A/G]G GGT & \\
\hline \hline
\end{tabular}

The first PCR mix included $1 \mu \mathrm{l}$ each of primers MSP-1, MSP-2A, and MSP-2B with $3 \mu$ l of the template DNA. The second nested reaction contained a mixture of $1 \mu \mathrm{l}$ each of MSP-3, MSP-4A, and MSP-4B and $1 \mu \mathrm{l}$ of the first amplification reaction mixture. On $2 \%$ gel electrophoresis the assumed amplicon size for the MSP-3 and MSP-4B (Ent. bieneusi) product was $500 \mathrm{bp}$, and nearly $300 \mathrm{bp}$ for the MSP-3 and MSP-4A (Enc. and other species). Amplifications were done for primary and nested reactions with a Thermo Scientific, Biometra thermocycler, which included at first a denaturation step for $5 \mathrm{~min}$ at $95^{\circ} \mathrm{C}$ and 36 cycles of denaturation for $30 \mathrm{sec}$ at $95^{\circ} \mathrm{C}$, primer annealing at $55^{\circ} \mathrm{C}$ for $1 \mathrm{~min}$, and elongation at $72^{\circ} \mathrm{C}$ for $2 \mathrm{~min}$. The final elongation step reached to $10 \mathrm{~min}$ hold cycle at $4^{\circ} \mathrm{C}$. This contained the positive control DNA extract of Ent. bieneusi and Enc. intestinalis both provided by Dr. Spencer Polley from England and by Dr. Karim Aoun from Tunisia.

RFLP-PCR protocol performed according to Katzwinkel-Wladarsch et al. $^{[15]}$ for all positive nested PCR samples used Fast Digest MnlI restriction enzyme (Fermentas life sciences \#FD1074). The PCR micro tubes were incubated for $5 \mathrm{~min}$ in a heat block at $37^{\circ} \mathrm{C}$. This was followed by heating the enzyme for $5 \mathrm{~min}$ at $65^{\circ} \mathrm{C}$ for inactivation. Finally, RFLP-PCR products were observed by agarose gel electrophoresis.

For DNA sequencing, PCR products of the second nested PCR reaction mixture were refined using 
QIAquick PCR Purification Kit (Qiagen, Valencia, CA, USA) and the eluted DNA was stored at $-20^{\circ} \mathrm{C}$. Nucleotide sequencing was done using BigDye Terminator v3.1 Cycle Sequencing Kit (Cat. NO.: 4337455, USA). The BigDye X Terminator TM purification kit (Cat. NO.: 4376486, USA) was used for purification of labeled extension products from excess dye terminators. Finally, establishment of DNA sequence was done by DNA analyzer (Applied Biasystems, California 94404, USA). The primers for sequencing were MSP-3, MSP$4 \mathrm{~A}$, and MSP-4B and each amplicon was sequenced in the two directions ${ }^{[14]}$.

Statistical analysis: Data analyzing was performed by Chi-square test; a $P$ value $<0.05$ was considered statistically significant. For comparative evaluation of the three chosen techniques; staining, nested PCR and RFLP PCR, presence of microsporidia by two or more techniques was considered as true positive. Absence of microsporidia by all the three techniques was considered true negative. Sensitivity, specificity, positive predictive value, negative predictive values as well as diagnostic accuracy were calculated as per standard formulae.

Ethical consideration: The study protocol was approved in August 2016 by the Institutional Ethics Committee of Faculty of Medicine, Ain Shams University, Egypt, together with other ethical statements for human consents and treatment of infected patients.

\section{RESULTS}

By microscopic examination of WMT and MZN stained smears, microsporidial spores appeared as pink or reddish oval bodies against a light green background by both stains. Spores ranged from $0.97-1.34 \mu \mathrm{m}$ in width, and 2.19-2.56 $\mu \mathrm{m}$ in length (Fig. 1).

The age range for the selected 323 subjects was between 2-68 years with mean age of $37.7 \pm 15.1$; 149 (46.1\%) were males and 174 (53.9\%) were females. There was no statistical significance between microsporidiosis and age and sex $(P=0.12)$.
The recorded general prevalence of microsporidiosis as determined by examination of stained smears, was $45 / 323$ (13.9\%); where $25 / 173$ (14.5\%) were immune compromised, and 20/150 (13.3\%) were immune competent. Out of the 45 positive cases in the two groups the percentage was $25 / 45$ (55.6\%) in immune compromised, and 20/45 (44.4\%) in immune competent groups, with no statistical difference between the two groups (Table 1). Positivity in subgroups I and II recorded 15/173 (8.7\%) in symptomatic group Ia, 10/173 (5.8\%) in asymptomatic group Ib. In symptomatic and asymptomatic groups IIa and IIb, positivity was 10/150 (6.7\%) for both. Of the total 45 positive cases, $15 / 25(60 \%)$ of group I, and $10 / 20(50 \%)$ of group II were symptomatic with no statistical significance (Table 1). Complaints of symptomatic cases in the 45 positive cases were shared in some cases and included diarrhea 23/45 (51.1\%), abdominal pain $15 / 45$ (33.3\%), distension $14 / 45$ (31.1\%), maldigestion 10/45 (22.2\%) and weight loss $10 / 45(22.2 \%)$. By exclusion, 20 cases $(44.4 \%)$ were asymptomatic with statistically significance difference $(P<0.001)$ (Table 1$)$. History of animal contact was noted in 9/45 (20\%) of positive cases in groups I and II; $1 / 25(4 \%)$ immune compromised (insignificant, $P=$ $0.16)$ and $8 / 20(40 \%)$ immune competent positive cases (significant, $P<0.01$ ) (Table 1 ).

Etiological distribution in the 25 positive immune compromised patients in group I showed that $3(12 \%)$ had autoimmune diseases, $4(16 \%)$ had renal failure, $2(8 \%)$ had leukemia, $5(20 \%)$ were subjected to chemotherapy and $4(16 \%)$ to radiotherapy, $4(16 \%)$ were elderly, 3 (12\%) had lymphoma, and 2 (2\%) had underwent renal transplantation. Some patients had more than one of these causes for their compromised immune state (blended etiology). As shown from table (2), the relation between microsporidiosis and the immune status of the individuals or etiology of immune compromised condition was insignificant $(P>0.05)$.

For the molecular assays, nested-PCR confirmed microsporidiosis in all of the $25(100 \%)$ microscopically positive samples of group I. Out of the total 45 microscopically positive patients in the
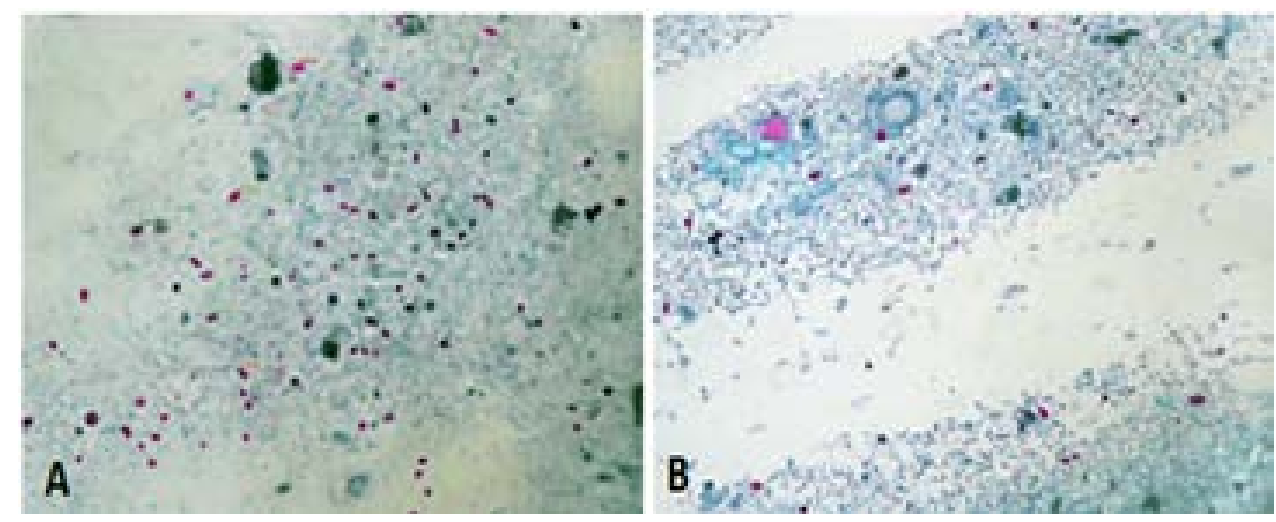

Fig. 1: Microsporidial spores stained by (A): modified trichrome stain, x1000 magnification, (B): modified Ziehl-Neelsen stain, x1000 magnification. 
two groups 44/323 (13.6\%) were confirmed positive using PCR (Table 3). Ent. bieneusi reacted at $500 \mathrm{bp}$ and Enc. intestinalis at $300 \mathrm{bp}$ molecular weight (MW), as compared to reference strains from England and Tunisia (positive control) (Fig. 2). In group I patients, Ent. bieneusi, Encephalitozoon spp. and mixed infection with both were identified in 5 (20\%), 5 (20\%) and 15 $(60 \%)$ samples respectively. In group II, 19/20 (95\%) microscopically positive samples included 6 (31.6\%) infected with Ent. bieneusi only, 3 (15.8\%) infected with Encephalitozoon species only and 10 (52.6\%) individuals had mixed infection with both. There was no statistical significance for presence of certain species among the immune compromised group I patients and the immune competent group II individuals (Table 4).

In RFLP PCR procedure, MnlI restriction enzyme cut the Ent. bieneusi DNA into bands with different MWs of 20, 30, 50, 60, 80, 90 and 180 bp. The Enc. intestinalis DNA was cut into bands with MWs of 20, 30, 60 and

Table 1: Relations between microsporidiosis and different variables in the studied groups.

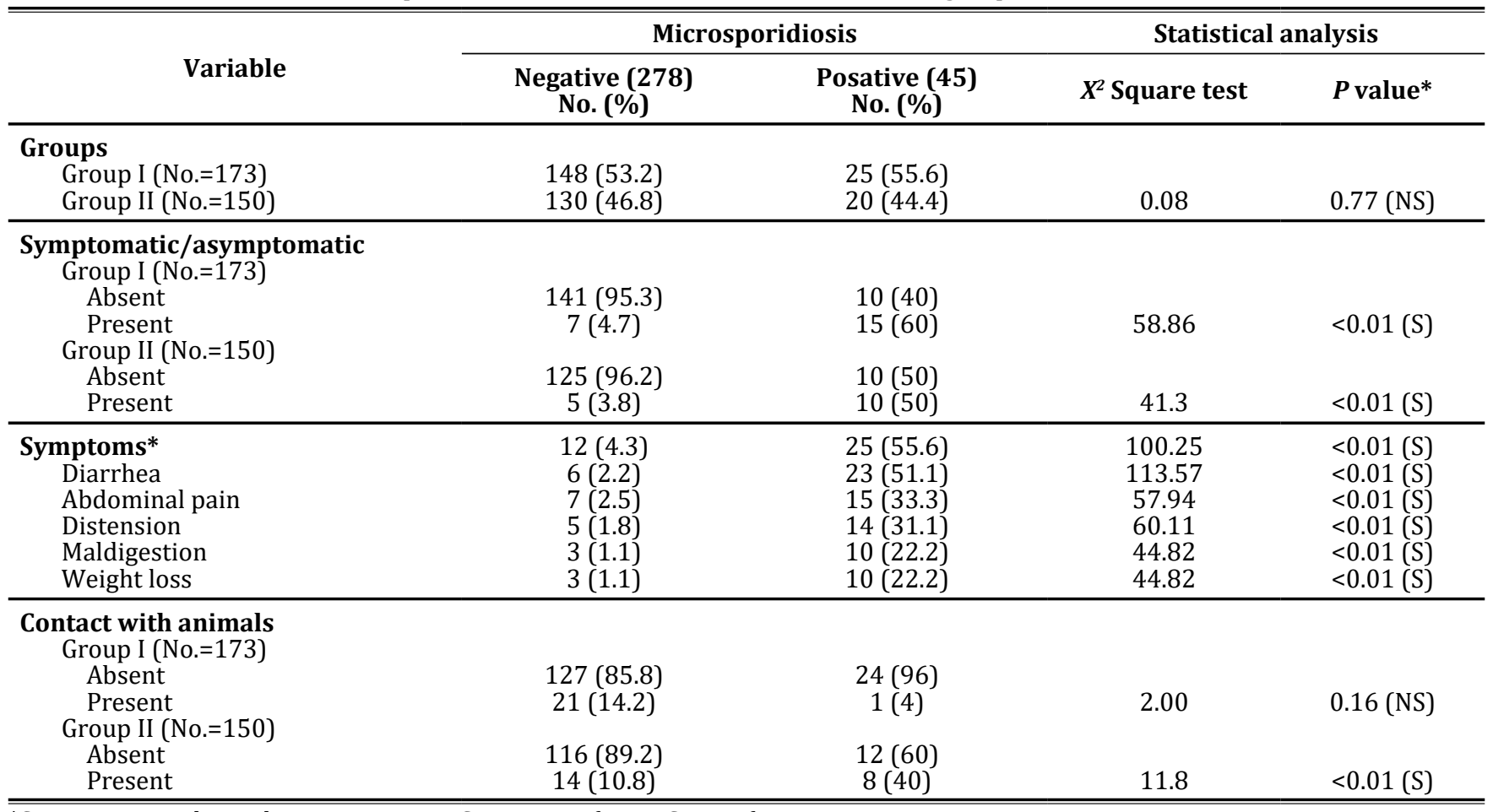

*Symptoms overlapped in some cases, NS: non-significant, S: significant.

Table 2: Relation between microsporidiosis and etiology among immune compromised patients in group I.

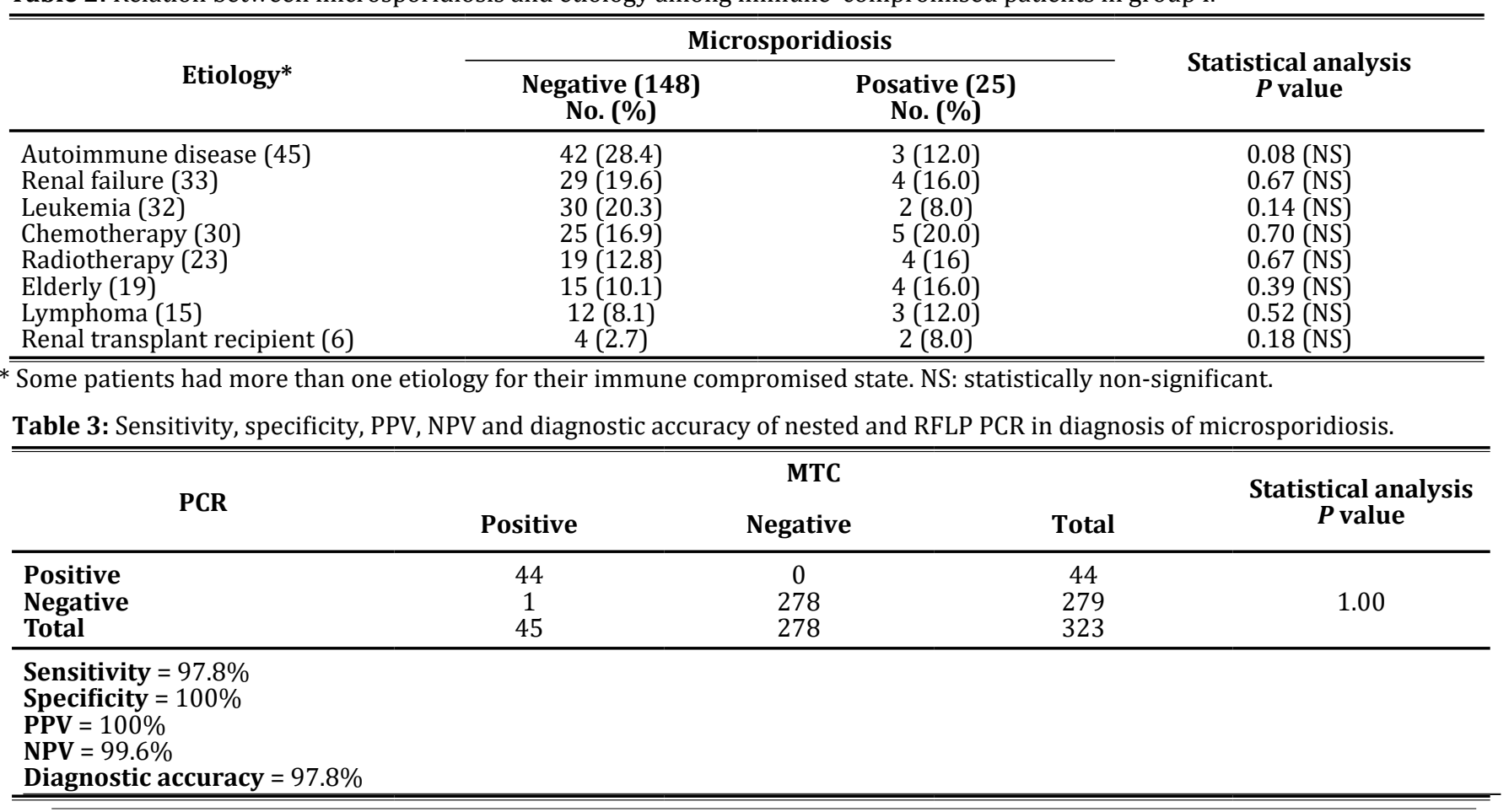


Table 4: Percentages of infection with Ent. bieneusi alone, Encephalitozoon species alone and mixed infection with both species by nested PCR in studied groups

\begin{tabular}{lcccc}
\hline \hline & $\begin{array}{c}\text { Group I (No.=25) } \\
\text { Immunocompromised }\end{array}$ & $\begin{array}{c}\text { Group II (No.=20) } \\
\text { Immuncompetent }\end{array}$ & \multicolumn{2}{c}{$\begin{array}{c}\text { Statistical } \\
\text { analysis }\end{array}$} \\
\cline { 2 - 5 } & No. (\%) & No. (\%) & Test value & P value \\
\hline Ent. bieneusi only & $5(20)$ & $6(31.6)$ & $0.77^{*}$ & 0.38 (NS) \\
Encephalitozoon species only & $5(20)$ & $3(15.8)$ & $0.13^{* *}$ & $1.0(\mathrm{NS})$ \\
Mixed infection & $15(60)$ & $10(52.6)$ & $0.24^{*}$ & 0.63 (NS) \\
\hline
\end{tabular}

*Chi square test, ${ }^{* *}$ Fisher exact test. $P$ value $>0.05$ is statistically non-significant (NS).
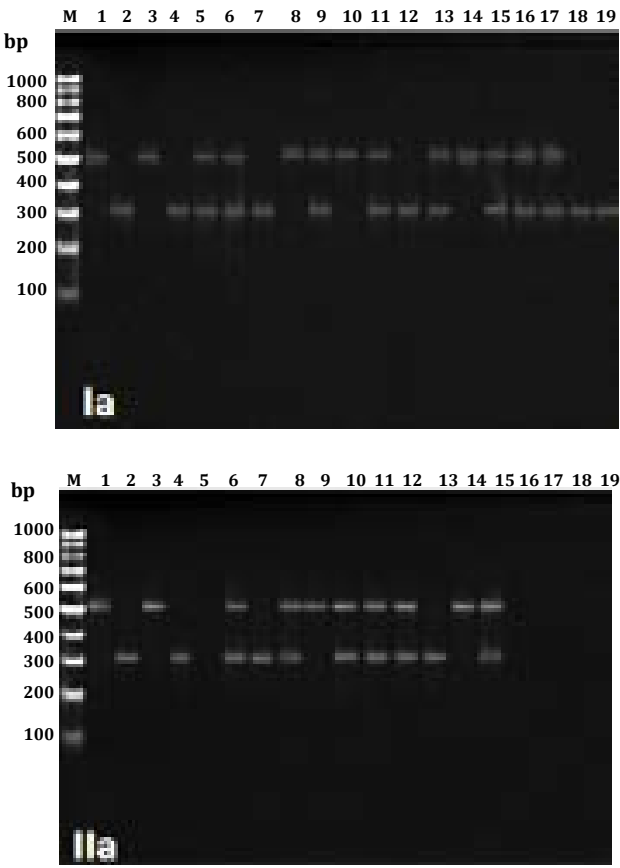
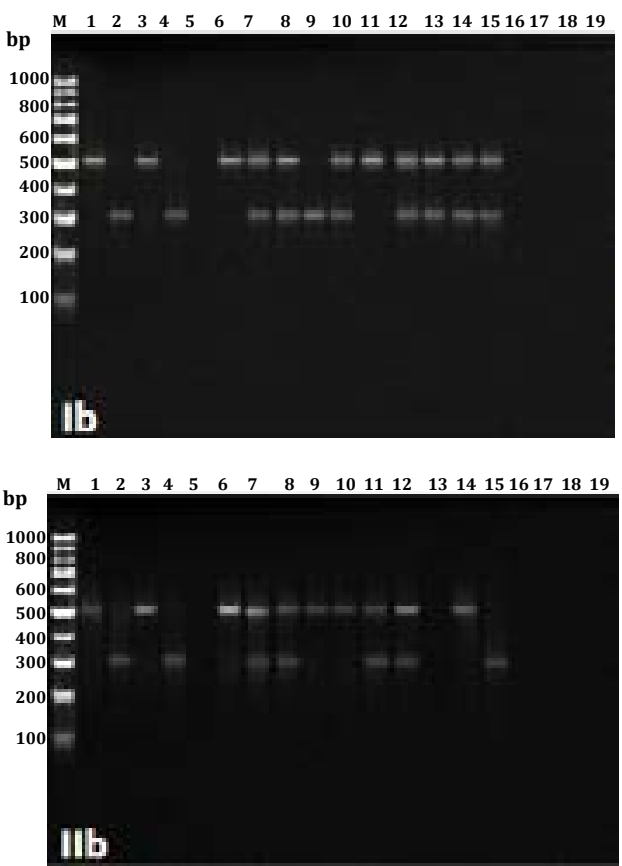

Fig. 2. Nested PCR showed Ent. bieneusi bands at 500 bp and Enc. intestinalis bands at 300 bp molecular weight in comparison to reference strains from England and Tunisia (Positive control).

Lane M: DNA molecular marker (100 - 1000 bp). Lane 1: Positive control for Ent. bieneusi. Lane 2: Positive control for Enc. intestinalis (Positive control in lanes 1 and 2 were provided from England). Lane 3: Positive control for Ent. bieneusi. Lane 4: Positive control for Enc. intestinalis. (Positive control in lanes 3 and 4 were provided from Tunisia). Group Ia: lanes 8, 10, 14: Ent. bieneusi; lanes 7, 12, 18, 19: Enc. intestinalis; lanes 5, 6, 9, 11, 13, 15, 16, 17: mixed infection (Ent. bieneusi and Enc. intestinalis). Group IIa: lanes 9, 15: Ent. bieneusi; lanes 7, 13: Enc. intestinalis; lanes 6, 8, 10, 11, 12, 14: mixed infection. Group Ib: lane 6: Ent. bieneusi; lane 9: Enc. intestinalis; lanes 7, 8, 10, 11, 12, 13, 14, 15: mixed infection. Group IIb: lanes 6,9,10,14: Ent. bieneusi; lane 15: Enc. intestinalis; lanes 7,8,11,12: mixed infection.

160 bp. The Enc. cuniculi DNA was cut into bands with MWs of 90 and $210 \mathrm{bp}$ (Fig. 3). RFLP-PCR confirmed all of the nested PCR 44/45 (97.8\%) positive samples in the two groups. The sensitivity and specificity of both nested and RFLP PCR were $97.8 \%$ and 100\%, respectively, with positive predictive value (PPV) $100 \%$, negative predictive value (NPV) $99.6 \%$, and diagnostic accuracy $97.8 \%$ (Table 4).

Relation between animal contact and different species of microsporidia detected by RFLP PCR showed statistically significant $(P=0.03)$ result of $36.4 \%$ only with Ent. bieneusi infection. A significant difference was observed between presence of symptoms and different species of microsporidia except for Enc. cuniculi spp. detected by RFLP PCR (Table 5).

Five nested PCR products (MSP $-1,-3,-4$, and MSP-2B, MSP-4B) with single species infection were purified and subjected to sequencing and compared to the sequences published on the Blast website to obtain the different genotypes and their related importance in pathogenicity and symptom production Accordingly Ent. bieneusi genotype D was detected in a renal transplant recipient patient in group Ia with $100 \%$ identity with the published blast sequence of Ent. bieneusi genotype D under accession number (AF101200) (Fig. 4A). Ent. bieneusi genotype K was detected in two patients; one in group Ib, and the other in group IIb. Blast alignment gave $98 \%$ and 100\% identity with the published genotype $\mathrm{K}$ blast sequence under accession number (AF267141) respectively (Fig. 4B, 4C). Ent. bieneusi sequence for genotype B was detected in group IIa with no history of animal contact, suffering from diarrhea, abdominal pain, distension, mal digestion and weight loss, with $100 \%$ identity to the published Ent. bieneusi genotype $\mathrm{B}$ blast sequence under accession number (AF101198) (Fig. 4D). A sequence of Enc. intestinalis belonging to a 26 years old symptomatic patient on corticosteroid therapy due to autoimmune disease, with no history of animal contact, suffering from abdominal pain and distension, gave 99\% identity with the published blast sequences under accession number (AB897500) (Fig. 4E). 
PARASITOLOGISTS UNITED JOURNAL
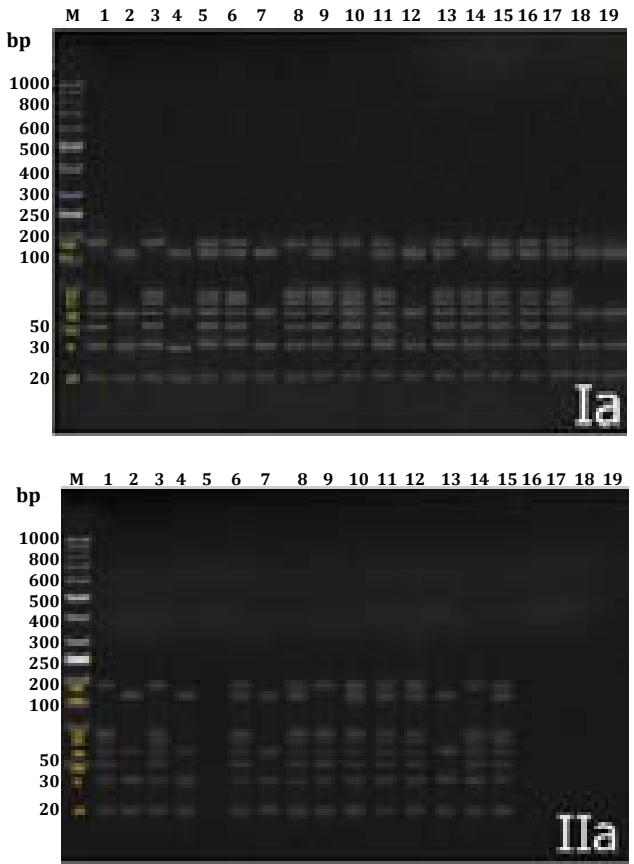
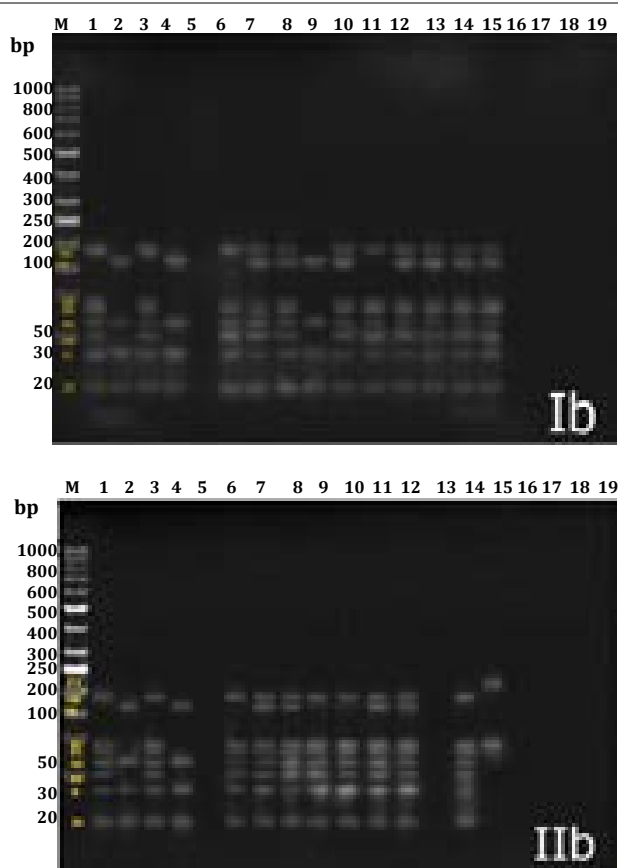

Fig. 3. RFLP PCR showed Ent. bieneusi bands at 20, 30, 50, 60,80, 90, 180 bp, Enc. intestinalis bands at 20, 30, 60, 160 bp, and Enc. caniculi at 90,210 bp molecular weight, in comparison to positive controls.

Lane M: DNA molecular marker (50-1000 bp). Lane 1: Positive control for Ent. bieneusi. Lane 2: Positive control for Enc. intestinalis. (Positive control in lanes 1 and 2 were provided from England). Lane 3: Positive control for Ent. bieneusi. Lane 4: Positive control for Enc. intestinalis. (Positive control in lanes 3 and 4 were provided from Tunisia). Group Ia: lanes 8, 10, 14: Ent. bieneusi; lanes 7, 12, 18, 19: Enc. intestinalis; lanes 5, 6, 9, 11, 13, 15, 16, 17: mixed infection (Ent. bieneusi and Enc. intestinalis). Group IIa: lanes 9, 15: Ent. bieneusi; lanes 7, 13: Enc. intestinalis; lanes 6, 8, 10, 11, 12, 14: mixed infection. Group Ib: lane 6: Ent. bieneusi; lane 9: Enc. intestinalis; lanes 7, 8, 10, 11, 12, 13, 14, 15: mixed infection. Group IIb: lanes 6, 9, 10, 14: Ent. bieneusi; lane 15: Enc. intestinalis; lanes 7, 8, 11, 12: mixed infection.

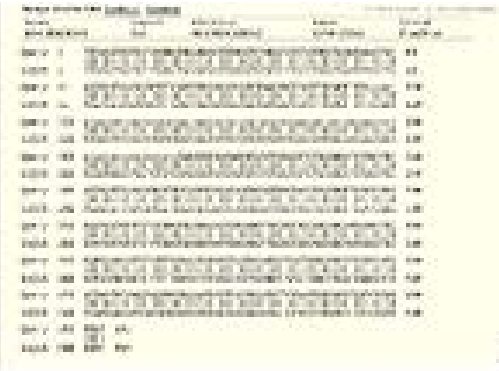

A

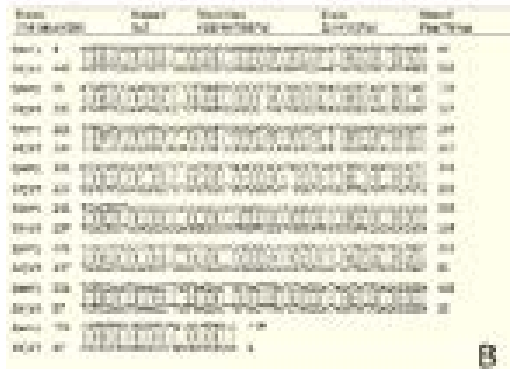

B
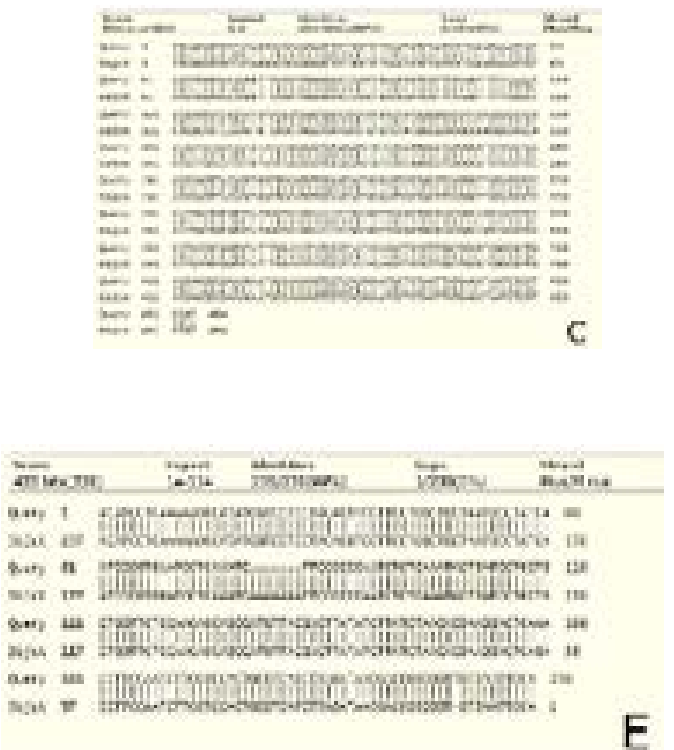

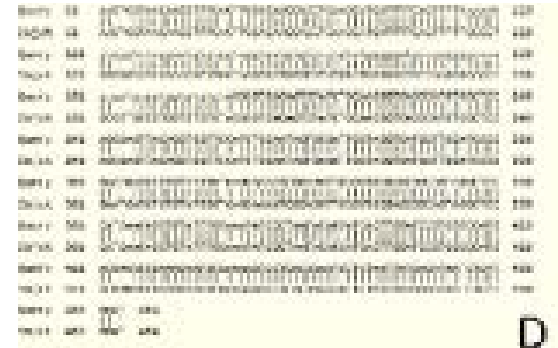

Fig. 4. DNA sequencing. A) Alignment on blast of Ent. bieneusi genotype D with $100 \%$ identity. B) Alignment on blast of Ent. bieneusi genotype K with $98 \%$ identity. C) Alignment on blast of Ent. bieneusi genotype K with $100 \%$ identity. D) Alignment on blast of Ent. bieneusi genotype B with $100 \%$ identity. E) Alignment on blast of Enc. intestinalis with $99 \%$ identity. 
Table 5: The relation between symptoms and animal contact with different microsporidia species detected by RFLP PCR.

\begin{tabular}{|c|c|c|c|c|}
\hline & \multirow{2}{*}{$\begin{array}{c}\text { Single species } \\
\text { No. }=18 \\
\text { No. }(\%)\end{array}$} & \multirow{2}{*}{$\begin{array}{c}\text { Mixed infection } \\
\text { No. }=25 \\
\text { No. }(\%)\end{array}$} & \multicolumn{2}{|c|}{ Statistical analysis } \\
\hline & & & $X^{2}$ square & $P$ value \\
\hline Occurrence of symptoms & $11(61.1 \%)$ & $14(56)$ & 0.112 & 0.73 \\
\hline Diarrhea & $11(61.1 \%)$ & $12(48)$ & 0.72 & 0.39 \\
\hline Abdominal pain & $7(38.9 \%)$ & $8(32)$ & 0.21 & 0.64 \\
\hline Distension & $7(38.9 \%)$ & $7(28)$ & 0.56 & 0.45 \\
\hline Maldigestion & $4(22.2 \%)$ & $6(24)$ & 0.01 & 0.89 \\
\hline Weight loss & $5(27.8 \%)$ & $5(20)$ & 0.35 & 0.55 \\
\hline Animal contact & $5(27.8 \%)$ & $5(20)$ & 0.35 & 0.55 \\
\hline
\end{tabular}

$P$ value $>0.05$ is statistically non-significant (NS).

\section{DISCUSSION}

Comparable morphological descriptions of microsporidia spores were reported using Weber's chromotrope-based stains. Spores appeared pinkish red and measured 0.9 to $1.2 \mu \mathrm{m}$ and 1 to $2 \mu \mathrm{m}$ in length, respectively with the characteristic posterior vacuole and belt-like stripe in the middle ${ }^{[3]}$. These intracellular protozoa are found in different organs of many wild and domestic animals ${ }^{[16]}$. Upgrading of diagnostic methods over the last 25 years guided the recognition of many novel species of microsporidia that spread from enteric to systemic sites of infection promoting the formation of scattered lesions ${ }^{[17]}$.

In the current study, 45/323 (13.9\%) of microsporidiosis were detected by microscopic examination of specifically stained smears using WMT and MZN stains including 25/173 (14.5\%) of the immune compromised patients and $20 / 150$ $(13.3 \%)$ of the immune competent individuals. Of these 44/323 (13.6\%) were confirmed positive using PCR, missing one case only. Similarly, Franzen and Müller ${ }^{[18]}$ detected negative PCR results for microscopically positive microsporidial samples. They attributed this to inhibitors of DNA extraction or prolonged storage of samples that lead to degeneration of the spores and their DNA. Sokolova et al. ${ }^{[14]}$ detected microsporidia in stool smears stained by modified trichrome blue and PCR in $18.9 \%$ of specimens from Russia. Moreover, in another study performed in Iran, stool specimens examined by direct wet smear, Ziehl-Neelsen and modified trichrome blue stains revealed microsporidia spores in $18.5 \%$ of samples ${ }^{[19]}$. Parallel results of a study using regular PCR technique reported a detection rate of microsporidiosis of $17 \%$ in cancer patients in Egypt $^{[20]}$

Previous results reported a prevalence of $13.9 \%$ in HIV-positive patients ${ }^{[21]}$. Also, using trichrome staining of fecal smears or PCR, the occurrence of intestinal microsporidiosis was recorded in $12.5 \%$ of patients with impaired immunity due to various primary immunodeficiencies and/or immunosuppressive therapy, and in $16.7 \%$ of patients with medicallyinduced immune suppression (transplant recipients) ${ }^{[22]}$. Liu et al. $^{[23]}$ observed that the infection rate of Ent. bieneusi was $11.6 \%$ in HIV-positive patients, and it was not found in HIV-negative patients by PCR. A higher incidence, $23.3 \%$ was reported using WMT stain in 23.3 $\%$ of fecal samples from HIV-infected patients ${ }^{[24]}$. Lobo et al. ${ }^{[21]}$ reported a prevalence of $8.5 \%$ in HIV-negative patients, while Lono et al. ${ }^{[25]}$ reported a prevalence of $2.9 \%$ in stool samples from HIV-negative control group. As regards age range of selected cases, no statistically significant difference was detected $(P=0.12)$. This was also stated in a similar report, but dealing with HIVpositive patients, in whom the age range was between 20 to 60 years $^{[14]}$. In addition, Lui et al. ${ }^{[23]}$ observed no age or sex associated differences in microsporidial positive patients in their study. Conversely another previous study showed that the percentage of children who proved to be microsporidia positive $(18.8 \%)$ was significantly higher than that of adults $(10.2 \%)$ ${ }^{[22]}$. In addition, the highest prevalence of unapparent microsporidial infection (24.2\%) in immune competent individuals with age range between 6 and 11 years old, and the lowest prevalence for individuals with age range between 12 to 19 years old (5.9\%) was reported ${ }^{[26]}$. However, Lono et al. ${ }^{[27]}$ reported that microsporidiosis occurred more in adults $(22.7 \%)$ than children (20\%), which was statistically insignificant in a community with ages ranging from 9 to 60 years.

In our study no statistically significant differences $(P=0.69)$ were observed as regards sex distribution. Similarly, previous studies did not find any statistically significant attribution for gender or racial bias concerning prevalence of microsporidiosis ${ }^{[25]}$. An additional study did not observe any statistically significant differences in gender of microsporidia positive and negative HIV-infected patients ${ }^{[14]}$.

The present work showed no statistical significant difference of microsporidiosis between immune compromised (55.6\%) and immune competent $(44.4 \%)$ positive patients $(P=0.77)$. In agreement, microsporidial parasites were found in both immune competent and immune compromised diarrheal subjects in Tunisia ${ }^{[4]}$; while a higher prevalence was previously reported among immune compromised children with malignant diseases $(25.56 \%)$ than in the healthy control group $(3.33 \%)^{[28]}$. Concerning the different etiologies predisposing for microsporidiosis in immune compromised positive cases, there was no 
statistical significant difference between the infection and the underlying etiology for compromised immunity. Also, Chabchoub et al. ${ }^{[4]}$ reported microsporidial infection in $11.8 \%$ and in $8.6 \%$ of HIV-infected patients and patients with myeloma, respectively.

In the present study diarrhea appeared to be the main complaint in positive symptomatic patients. Out of the 25 immune compromised positive cases in group I, $15(60 \%)$ were symptomatic with complaints of: diarrhea (51.1\%), abdominal pain (33.3\%), abdominal distension (31.1\%), maldigestion and weight loss (22.2\%); where all symptoms were statistically significant $(P<0.01)$. Ojuromi et al. ${ }^{[24]}$, reported a clear association of $95 \%$ between microsporidia and diarrhea. Moreover, El-Sobky and El-Nahas ${ }^{[20]}$ reported microsporidia in a diarrheic group (23\%) more than in a non-diarrheic group (5.7\%). Concerning the other symptoms higher positive results of $80 \%$ were reported by Aikawa et $a l^{[29]}$ among rheumatic patients who complained of diarrhea (29\%), abdominal pain (32\%) and weight loss (6\%). Out of the 20 immune competent individuals in our study, $50 \%$ were symptomatic with various complaints, and the other $50 \%$ had no apparent gastrointestinal complaints. It is noteworthy that microsporidial spores were often identified in formed stool samples $(10.9 \%)^{[26]}$.

Using PCR a higher incidence was previously reported where diarrhea was recorded in $92.3 \%$ of HIV microsporidia positive patients together with weight loss in $94.5 \%{ }^{[30]}$. Statistical significant lower incidences of diarrhea were also reported in HIV-positive (21.6\%) and HIV-negative patients $(9 \%)^{[31]}$. Other authors stated that although diarrhea and malabsorption seemed to be the most common clinical problems associated with microsporidial infections, namely, Ent. bieneusi, there were no statistically significant associations between the presence of microsporidial spores in fecal specimens and patients with diarrhea ${ }^{[32]}$.

In the current work, there were no statistically significant differences for occurrence of Ent. bieneusi, Encephalitozoon species and mixed infection between patients of group I and II with $P$ values $0.38,1$ and 0.63 , respectively. Similarly, it was reported that Ent. bieneusi, Enc. intestinalis, and both species were insignificantly identified in immune compromised cases including malignancy, patients receiving cytotoxic drug therapy or not, patients under corticosteroid therapy for more than 6 months, end stage renal failure on dialysis, splenectomized patients and diabetics ${ }^{[33]}$.

The prevalence of Ent. bieneusi, Enc. intestinalis and mixed infection by both species in the 25 immune compromised group I patients were $20 \%, 20 \%$ and $60 \%$, respectively. A study done on HIV-infected patients showed a higher prevalence of Enc. intestinalis $12.8 \%$, than that of Ent. bieneusi $1.2 \%{ }^{[14]}$. Moreover, reported prevalence of Ent. bieneusi, Vittaforma-like species and co-infection with both species was $7 \%$, $8.2 \%$ and $1.2 \%$ respectively, in fecal specimens of HIV positive patients ${ }^{[21]}$.

Our study did not include any HIV patients, simply because they were not available. We recorded a statistically insignificant $(P>0.05)$ association of different microsporidia species with specific disease or state of compromisedimmunity, exceptforinfection with Enc. intestinalis which showed statistical significance $(P<0.01)$ in association with renal transplant recipient patients. On the other hand there was no significant difference for presence of certain microsporidial species in leukemic patients. A previous study stated that the percentage of microsporidiosis using PCR among leukemic patients $(28 / 100)$ was higher than that in other groups of cancer patients, where Enc. intestinalis was the only species detected in positive stool samples ${ }^{[20]}$. Regarding Ent. bieneusi genotype B, it was detected in the present study with $100 \%$ identity with that found in blast sequence in a symptomatic immune competent patient suffering from diarrhea, abdominal pain, distension, maldigestion and weight loss, and with no history of animal contact. Genotype $B$ is considered as an anthroponotic genotype specially found in HIV positive patients ${ }^{[34-36]}$ and as the dominant anthroponotic genotype in developed countries ${ }^{[23]}$. On the contrary, another study reported genotype $\mathrm{B}$ in both HIV-seropositive and HIV-seronegative populations ${ }^{[31]}$.

As regards infection with Ent. bieneusi and animal contact we recorded a significant statistical difference $(P=0.03)$. Researchers stated that molecular analysis of the 243-bp internal transcribed spacer of the rRNA gene showed significant genetic variability among Ent. bieneusi isolates, which supports the zoonotic transmission of some of its genotypes from animals ${ }^{[37]}$. Others revealed that animals may represent a major source for spore transmission of opportunistic infection in humans ${ }^{[38]}$.

Genotype D was detected in the present study in a symptomatic immune compromised case with history of animal contact. In Tunisia, four isolates were found identical to the published Ent. bieneusi ITS sequences of genotype $\mathrm{D}^{[39]}$. In China, genotype $\mathrm{D}$ was the most prevalent genotype discovered in 17 samples from symptomatic children, HIV-positive patients, and HIVnegative individuals. Furthermore, genotype $\mathrm{D}$ was observed in not less than 15 animal species, river water and wastewater ${ }^{[40]}$. Our results agree with other studies that reported the zoonotic potential of genotype $\mathrm{D}^{[32,40,41]}$, and that it is a non host specific genotype with a broad host and geographic range ${ }^{[42,43]}$. Genotype K of Ent. bieneusi was also recorded in our study in 2 cases; an immune compromised asymptomatic patient with negative animal contact and in an immune competent asymptomatic individual with positive animal contact. Similar results were reported where genotypes D and K were the most prevalent genotypes and indicating that 
genotype $\mathrm{K}$ was significantly associated with diarrhea among HIV-positive patients ${ }^{[34,35,44]}$. Consequently the broad host and geographic range of genotype $\mathrm{K}$ was validated $^{[43]}$.

Regarding Enc. intestinalis, the present study detected only one sequence with $99 \%$ identity to Enc. intestinalis published blast sequences in a symptomatic immune compromised patient on corticosteroid therapy complaining of abdominal pain and distension with negative history for animal contact. This same species was reported in a patient with an unconfirmed HIV status who presented with diarrhea ${ }^{[14]}$. It was also reported in stool specimens from 2 patients (1 HIV patient, 1 patient with myeloma), where the two amplicons had $96 \%$ and $99 \%$ sequence identity with Enc. intestinalis (Genbank accession no. EU436735) ${ }^{[4]}$.

Conclusion: Prevalence of intestinal microsporidiosis was slightly higher in symptomatic immune compromised than immune competent populations, in whom diarrhea was the provoking symptom. Both staining and PCR techniques detected nearly the same result. As regards microsporidia species, a slightly higher prevalence of Ent. bieneusi than of Enc. intestinalis was observed among studied groups. No relation was observed between etiology of compromised immunity and microsporidiosis. History of animal contact was statistically significant among immune competent group suggesting the zoonotic source for microsporidiosis. As regards microsporidia species, a slightly higher prevalence of Ent. bieneusi than of Enc. intestinalis was observed among studied groups. The recent application of PCR-based molecular methods helped reveal the distribution of genotypes of Ent. bieneusi. All genotyping studies performed until now were based on the analysis of the ITS sequences. Although some Ent. bieneusi genotypes in the present study were found to be related to animal contact and human infection, this can't be accurately defined due to the limited number of available sequences.

Limitations and recommendations: Diagnosis of microsporidiosis requires more than one stool examination by an experienced parasitologist because of its small size and difficulty in recognition by microscopic examination. The identification of the risk factors as immune incompetence and animal contact associated with microsporidiosis, in addition to molecular diagnosis of microsporidiosis, will help in determining the distribution of Ent. bieneusi genotypes by geographical location, to improve source tracking, and to calculate the host range and pathogenic potential of an isolate. To determine the zoonotic nature of microsporidiosis, further studies should be done especially for patients who are in direct contact with animals as veterinarians, pet owners, shepherds, as well as workers in poultry breeding and slaughter houses to determine what type of animal is responsible for microsporidiosis.
Acknowledgments: This work was developed at the Parasitology Research and Diagnostic Laboratory Unit, Medical Parasitology Department and at Professor Dr. Ali Khalifa Cancer Detection Unit, Biochemistry department, Faculty of Medicine, Ain Shams University. We would like to thank the Radiotherapy, Chemotherapy, Hematology, Nephrology, Geriatrics Departments and Pediatric hospital, Faculty of Medicine, Ain Shams University for providing us with the patients' samples. Sincere thanks to Prof. Dr. Spencer Polley from England and Prof. Dr. Karim Aoun from Tunisia for their generous help.

Author contribution: Abd-Elbaki MH shared in choosing the aim of study, designing the plan of work and revising the manuscript. Arafa MA revised the manuscript, Abd-El Hameed DA conceived and designed the plan of work, analyzed the data, wrote and revised the manuscript. Habib KS revised the manuscript. Abdel Rahman AA shared in specimen collection, staining techniques, analyzing the data, writing and revising the manuscript and Anwar MM performed staining, PCR techniques and genotyping.

Conflict of interest: There is no conflict of interest

\section{REFERENCES}

1. Yang $\mathrm{H}, \mathrm{Mi} \mathrm{R}$, Cheng $\mathrm{L}$, Huang $\mathrm{Y}, \mathrm{An} \mathrm{R}$, Zhang $\mathrm{Y}$, et al. Prevalence and genetic diversity of Enterocytozoon bieneusi in sheep in China. Parasit Vectors 2018; 11: 587- 596.

2. Kim K, Yoon S, Cheun H, Kim J, Sim S, Yu J. Detection of Encephalitozoon spp. from human diarrheal stool and farm soil samples in Korea. J Korean Med Sci 2015; 30(3): 227-232.

3. Galván AL, Sánchez AM, Valentín MA, Henriques-Gil N, Izquierdo F, Fenoy S, et al. First cases of microsporidiosis in transplant recipients in Spain and review of the literature. J Clin Microbiol 2011; 49: 1301-1306.

4. Chabchoub N, Abdelmalek R, Mellouli F, Kanoun F, Thellier M, Bouratbinen A, et al. Genetic identification of intestinal microsporidia species in immune compromised patients in Tunisia. Am J Trop Med Hyg 2009; 80 (1): 24-27.

5. Saigal K, Khurana S, Sharma A, Sehgal R, Malla N Comparison of staining techniques and multiplex nested PCR for diagnosis of intestinal microsporidiosis. Diagn Microbiol Infect Dis 2013; 77: 248-249.

6. Ghoshal U, Khanduja S, Agarwal V, Dhole TN, Ghoshal UC. Comparative evaluation of staining techniques and polymerase chain reaction for diagnosis of intestinal microsporidiosis in immune compromised patients. Trop Parasitol 2015; 5(2): 101-105.

7. Nooshadokht M, Sharifi I, Mohammadi MA, Pirestani M Afgar A, Mahootchi A et al. Intestinal microsporidiosis in Iran: infection in immune-compromised and immune competent patients. Curr Med Mycol 2017; 6:30-36. 
8. Amer S, Kim S, Han J, Na K. Prevalence and genotypes of Enterocytozoon bieneusi in wildlife in Korea: a public health concern. Parasit Vectors 2019; 12: 160-166.

9. Conteas CN, Berlin OG, Ash LR, Pruthi JS. Therapy for human gastrointestinal microsporidiosis. Am J Trop Med Hyg 2000; 63: 121-127.

10. Han, B and Weiss, LM. Therapeutic targets for the treatment of microsporidiosis in humans. Expert Opin Ther Targets 2018; 22(11): 903-915.

11. Shane AL, Mody RK, Crump JA, Tarr PI, Steiner TS, Kotloff $\mathrm{K}$ et al. Infectious diseases society of American clinical practice guidelines for the diagnosis and management of infectious diarrhea. Clin Infect Dis 2017; 65: e45-80.

12. Weber R, Bryan RT, Owen RL, Wilcox CM, Gorelkin L, Visvesvara GS. Improved light-microscopical detection of Microsporidia spores in stool and duodenal aspirates. The Enteric Opportunistic Infections Working Group. N Engl J Med 1992; 326: 161-166.

13. World Health Organisation. Section I: Techniques of collection, preparation and examination of samples; permanent staining techniques. In Basic laboratory methods in Medical Parasitology 1991; 17-18.

14. Sokolova OI, Demyanov AV, Bowers LC, Didier ES, Yakovlev, AV, Skarlato SO, et al. Emerging microsporidian infections in Russian HIV-infected patients. J Clin Microbiol 2011; 49(6): 2102-2108.

15. Katzwinkel-Wladarsch S, Lieb M, Heise W, Loscher T, Rinder H. Direct amplification and species determination of microsporidian DNA from stool specimens. Trop Med Int Health 1996; 1 (3): 373-378.

16. Adesiji YO, Oloke JK. Challenges of the control of opportunistic infections of zoonotic origin in HIV/AIDS patients. Int J Immunol 2015; 3(2-1): 1-7.

17. Didier ES, Weiss LM. Microsporidiosis: Not just in AIDS patients. Curr Opin Infect Dis 2011; 24(5): 490-495.

18. Franzen C, Muller A. Molecular techniques for detection, species differentiation and phylogenetic analysis of microsporidia. Clin Microbiol Rev 1999; 12(2): 243-85.

19. Khalili B, Imani R, Boostani S. Intestinal parasitic infections in chronic psychiatric patients Sina Hospital Shahre-cord, Iran. Jundishapur J Microbiol 2013; 6(3): 252-255.

20. El Sobky MM, El Nahas NS. Detection and differentiation between Enterocytozoon bieneusi and Encephalitozoon intestinalis species in cancer patient's stools using PCR compared with different staining methods. PUJ 2012; 5(1): 19-26.

21. Lobo ML, Xiao L, Antunes F, Matos O. Microsporidia as emerging pathogens and the implication for public health: a 10-year study on HIV-positive and negative patients. Int J Parasitol 2012; 42: 197-205.

22. Bednarska M, Bajer A, Siński E, Wolska-Kuśnierz, B, Samoliński, B, Graczyk, TM. Occurrence of intestinal microsporidia in immunodeficient patients in Poland. Ann Agric Environ Med 2014; 21(2): 244-248.

23. Liu H, Jiang Z, Yuan Z, Yin J, Wang Z, Yu B, et al. Infection by and genotype characteristics of Enterocytozoon bieneusi in HIV/AIDS patients from Guangxi Zhuang autonomous region, China. BMC Infect Dis 2017; 17:684-691.
24. Ojuromi OT, Izquierdo F, Fenoy S, Fagbenro-Beyioku A, Oyibo W, Akanmu A, et al. Identification and characterization of microsporidia from fecal samples of HIV-positive patients from Lagos, Nigeria. PLoS One 2012; 7(4): 1-7.

25. Lono A, Kumar GS, Chye TT. Detection of microsporidia in local HIV-positive population in Malaysia. Trans $\mathrm{R}$ Soc Trop Med Hyg 2011; 105: 409-413.

26. Sak B, Brady D, Pelika nova M, Kve ton ova D, Rost M, Kostka M, et al. Unapparent microsporidial infection among immune competent humans in the Czech Republic. J Clin Microbiol 2011; 49(3): 1064-1070.

27. Lono A, Kumar GS, Chye TT. Prevalence of microsporidia in an indigenous Orang Asli community in Pahang, Malaysia. Trans R Soc Trop Med Hyg 2010; 104: 214218.

28. Antonios SN, Tolba OA, Othman AA, Saad MA. A preliminary study on the prevalence of parasitic infections in immune compromised children. J Egypt Soc Parasitol 2010; 40(3): 617-630.

29. Aikawa NE, Twardowsky AO, Carvalho JF, Silva CA, Silva ILAF, Ribeiro ACM, et al. Intestinal microsporidiosis: a hidden risk in rheumatic disease patients undergoing anti-tumor necrosis factor therapy combined with disease-modifying anti-rheumatic drugs? Clinics (Sao Paulo) 2011; 66(7): 1171-1175.

30. Endeshaw T, Kebede A, Verweij JJ. Intestinal microsporidiosis in diarrheal patients infected with human immunodeficiency virus- 1 in Addis Ababa, Ethiopia. Jpn J Infect Dis 2006; 59(5): 306-310.

31. Samie A, Obi CL, Tzipori S, Weiss LM, Guerrant RL. Microsporidiosis in South Africa: PCR detection in stool samples of HIV-positive and HIV-negative individuals and school children in Vhembe district, Limpopo Province. Trans R Soc Trop Med Hyg 2007; 101(6): 547-554.

32. Matos O, Lobo ML and Xiao L. Epidemiology of Enterocytozoon bieneusi infection in humans. J Parasit Res 2012; 1-19.

33. Rayan HZ, Ismail OA, El-Hamshary EM, Ibrahim GH, Omar SM. Contribution of multiplex real time PCR for detection and differentiation of Enterocytozoon bieneusi and Encephalitozoon intestinalis in fecal samples of immune compromised patients. PUJ 2013; 6(1): 51-58.

34. Mathis A, Weber R. Deplazes P. Zoonotic potential of the microsporidia. Clin Microbiol Rev 2005; 18(3): 423-445.

35. Ten Hove RJ, Van Lieshout L, Beadsworth MB, Perez MA, Spee K, Claas EC, et al. Characterization of genotypes of Enterocytozoon bieneusi in immune suppressed and immune competent patient groups. J Eukaryot Microbiol 2009; 56(4): 388 -393.

36. Stark D, van Hal S, Barratt J, Ellis, J, Marriott D, Harkness J. Limited genetic diversity among genotypes of Enterocytozoon bieneusi strains isolated from HIV-infected patients from Sydney, Australia. J Med Microbiol 2009; 58: 355-357.

37. Thellier M, Breton J. Enterocytozoon bieneusi in human and animals, focus on laboratory identification and molecular epidemiology. Parasite 2008; 15: 349-358. 
38. Al-Sadi HI, Al-Mahmood SS. Pathology of experimental Encephalitozoon cuniculi infection in immune competent and immune suppressed mice in Iraq. Path Res Inter 2014; 2014, 857036, 6 pages.

39. Chabchoub N, AbdelMalek R, Breton J, Kanoun, F, Thellier M, Bouratbine A, et al. Genotype identification of Enterocytozoon bieneusi isolates from stool samples of HIV-infected Tunisian patients. Parasite 2012; 19 : 147-151.

40. Yu F, Li D, Chang Y, Wu Y, Guo Z, Jia L, et al. Molecular characterization of three intestinal protozoans in hospitalized children with different disease backgrounds in Zhengzhou, central China. Parasit Vectors 2019; 12(1): 543-552.

41. Leelayoova S, Subrungruang I, Suputtamongkol Y, Worapong J, Petmitr PC, Mungthin M. Identification of genotypes of Enterocytozoon bieneusi from stool samples from human immunodeficiency virus-infected patients in Thailand. J Clin Microbiol 2006; 44(8): 3001-3004.

42. Espern A, Morio F, Miegeville M. Molecular study of microsporidiosis due to Enterocytozoon bieneusi and Encephalitozoon intestinalis among human immunodeficiency virus-infected patients from two geographical areas: Niamey, Niger, and Hanoi, Vietnam. J Clin Microbiol 2007; 45(9): 2999-3002.

43. Akinbo FO, Okaka CE, Omoregie R, Dearen T, Leon ET, Xiao L. Molecular epidemiologic characterization of Enterocytozoon bieneusi in HIV-infected persons in Benin city, Nigeria. Am J Trop Med Hyg 2012; 86(3): 441-445.

44. Wang, L, Zhang H, Zhao X, Zhang L, Zhang G, Guo $\mathrm{M}$, et al. Zoonotic Cryptosporidium species and Enterocytozoon bieneusi genotypes in HIV-positive patients on antiretroviral therapy. J Clin Microbiol 2013; 51(2): 557-563. 